\title{
Aspectos metodológicos para la elaboración de videos docentes para su uso como objetos de aprendizaje modulares y reutilizables
}

\author{
Eva María Llera a , Ignacio Zabalza, Begoña Peña, Sergio Usón, Amaya Martínez, Luis \\ Miguel Romeo
}

Universidad de Zaragoza - Departamento de Ingeniería Mecánica, 50018 Zaragoza, España. ae-mail: ellera@unizar.es

\begin{abstract}
The objective of this work is to reflect on the main methodological aspects to take into account in the development of video teaching for use as modular and reusable learning objects. This reflection is one of the results of the work carried out by the authors in a teaching innovation project funded by the University of Zaragoza during the academic years 2015-16, 2016-17 and 2017-18. In this project, 25 educational videos under licenses have been created to improve the learning outcomes of five subjects in the field of thermodynamics and thermal engineering, currently taught at three different qualifications. Preparing educational videos requires different strategies compared with an in real time session and aspects such as the approach of the contents, the adaptation to the environment and technical quality influence the effectiveness as a learning tool by what must be taken into account in its development phase.
\end{abstract}

Keywords: instructional videos, active methodologies, engineering education

\section{Resumen:}

El objetivo de este trabajo es reflexionar sobre los principales aspectos metodológicos a tener en cuenta en el desarrollo de vídeos docentes para su uso como objetos de aprendizaje modulares y reutilizables.

Esta reflexión es uno de los resultados del trabajo desarrollado por los autores en un proyecto de innovación docente financiado por la Universidad de Zaragoza durante los cursos académicos 2015-16, 2016-17 y 2017-18. En este proyecto, se han creado 25 videos docentes bajo licencias para mejorar los resultados de aprendizaje de cinco asignaturas en el campo de la Termodinámica y la Ingeniería Térmica, que actualmente se imparten en tres titulaciones distintas.

Preparar vídeos docentes requiere estrategias diferentes a las de una clase en vivo y aspectos como el enfoque de los contenidos, la adecuación al medio y la calidad técnica influyen en la efectividad como herramienta de aprendizaje por lo que deben de ser tenidos en cuenta en su fase de desarrollo.

Palabras clave: vídeos docentes, metodologías activas, enseñanza de la ingeniería 


\section{Introducción}

Numerosos autores (Kearney et al, 2012; Stefanova, 2014) y otras experiencias documentadas (Zabalza et al., 2017) demuestran cómo el uso de videos docentes crea auténticas oportunidades de aprendizaje para los estudiantes y fomentan el rigor académico.

Willmot et al. (2011) manifiestan que incorporar videos otras actividades de aprendizaje centradas en el estudiante conlleva, entre otros, un aumento de la motivación de los estudiantes, una mejora en la experiencia de aprendizaje, calificaciones más altas, un mayor potencial de desarrollo para aprendizajes más profundos y el desarrollo de la autonomía del estudiante.

Además de dichos beneficios, disponer de videos docentes facilita la aplicación de otros métodos de enseñanza-aprendizaje como el modelo de aula invertida (Peña et al, 2017), en el que los estudiantes pueden asimilar los fundamentos de un tema a su ritmo permitiendo explorar el contenido con mayor profundidad durante la sesión de clase.

No obstante, la efectividad de estos videos en el proceso enseñanza-aprendizaje depende de numerosos aspectos, algunos relacionados con la predisposición del estudiante (Cole et al., 2017), otros con la habilidad del profesor (Sujo de Montes and Gonzales, 2000), y muchos inherentes a su elaboración entre los que destacan el enfoque de los contenidos, la adecuación al medio y la calidad técnica.

En esta contribución se presenta cómo se definieron estos últimos aspectos en la elaboración del conjunto de videos resultante de un proyecto de innovación docente de la Universidad de Zragoza.

El proyecto, financiado por la Universidad de Zaragoza durante los cursos académicos 2015-16, 2016-17 y 2017-18 tenía como principal objetivo aprovechar las oportunidades que ofrecen las nuevas tecnologías del aprendizaje y del conocimiento para mejorar el proceso de enseñanza-aprendizaje en asignaturas del ámbito de la termodinámica y la ingeniería térmica, que actualmente se imparten en la Escuela de Ingeniería y Arquitectura de la Universidad de Zaragoza.

A lo largo del proyecto de innovación, se han creado 25 videos docentes (Tabla 1) bajo licencias para mejorar los resultados de aprendizaje de 5 asignaturas en el campo de la Termodinámica y la Ingeniería Térmica, que actualmente se imparten en tres titulaciones distintas (Tabla 2).

Tabla 1. Temática (y número) de vídeos docentes utilizados en las asignaturas

\begin{tabular}{ll}
\hline \multicolumn{2}{c}{ Temas } \\
\hline Tutorial del programa “Engineering Equation Solver”-EES (3) & Ciclos de refrigeración (2) \\
\hline Propiedades de las sustancias puras (4) & Psicrometría (2) \\
\hline Modelo de gas ideal (2) & Método de diferencias finitas-MDF (2) \\
\hline
\end{tabular}

(cc) EY-NC-ND 2018, Universitat Politècnica de València

Congreso IN-RED (2018) 


\begin{tabular}{ll}
\hline Balance de energía en sistemas cerrados (1) & Intercambiadores de calor (2) \\
\hline Balance de masa y energía en volúmenes de control (2) & Convección forzada exterior (1) \\
\hline Ciclos de potencia con turbina de gas-Brayton (1) & Motores Térmicos (1) \\
\hline Ciclos de potencia con turbina de gas-Rankine (2) & \\
\hline
\end{tabular}

Tabla 2. Asignaturas seleccionadas para el uso de vídeos docentes

\begin{tabular}{|c|c|c|c|c|c|}
\hline Asignatura & Titulación & Curso & $\begin{array}{l}\text { Créditos } \\
\text { ECTS }\end{array}$ & $\begin{array}{l}\mathrm{N}^{0} \text { de } \\
\text { vídeos } \\
\text { usados }\end{array}$ & $\begin{array}{c}\mathrm{N}^{\mathrm{o}} \text { de } \\
\text { estudiantes }\end{array}$ \\
\hline $\begin{array}{l}\text { Termodinámica Técnica y } \\
\text { Fundamentos de Transmisión } \\
\text { de Calor }\end{array}$ & $\begin{array}{l}\text { Grado en Ingeniería } \\
\text { de Tecnologías } \\
\text { Industriales }\end{array}$ & $2^{\circ}$ & 6 & 19 & 70 \\
\hline $\begin{array}{l}\text { Termodinámica Técnica y } \\
\text { Fundamentos de Transmisión } \\
\text { de Calor }\end{array}$ & $\begin{array}{l}\text { Grado en Ingeniería } \\
\text { Mecánica }\end{array}$ & $2^{\circ}$ & 6 & 19 & 64 \\
\hline $\begin{array}{l}\text { Termodinámica Técnica y } \\
\text { Fundamentos de Transmisión } \\
\text { de Calor }\end{array}$ & $\begin{array}{l}\text { Grado en Ingeniería } \\
\text { Electrónica y } \\
\text { Automática }\end{array}$ & $2^{\circ}$ & 6 & 10 & 85 \\
\hline Ingeniería Térmica & $\begin{array}{l}\text { Grado en Ingeniería } \\
\text { de Tecnologías } \\
\text { Industriales }\end{array}$ & $2^{\circ}$ & 6 & 2 & 50 \\
\hline Máquinas y Motores Térmicos & $\begin{array}{l}\text { Grado en Ingeniería } \\
\text { Mecánica }\end{array}$ & $3^{\circ}$ & 6 & 2 & 70 \\
\hline
\end{tabular}

\section{Objetivos}

El objetivo de este trabajo es presentar los principales aspectos metodológicos a tener en cuenta en el desarrollo de vídeos docentes para su uso como objetos de aprendizaje modulares y reutilizables desde la propia experiencia de los autores.

Asimismo, a partir del análisis de los resultados obtenidos por una muestra de los estudiantes involucrados en el proyecto docente, se analiza si los aspectos inherentes a la elaboración de los videos contribuyen a su efectividad como herramientas de mejora del proceso de enseñanza-aprendizaje.

\section{Desarrollo de la innovación}

Los videos sobre los que se discute son el resultado de un proyecto de innovación docente de la Universidad de Zaragoza.

El objetivo de dicho proyecto, todavía en curso, es mejorar la eficiencia en el proceso de adquisición, por parte del alumnado, de las competencias específicas y los resultados de aprendizaje de cuatro asignaturas del ámbito de la Ingeniería Térmica, impartidas en la Escuela de Ingeniería y Arquitectura (EINA) a través del uso de videos docentes. 
Hasta el momento se han elaborado 25 videos que han sido utilizados en diversas actividades formativas de 5 asignaturas relacionadas con la termodinámica aplicada y para los que se ha medido a través de diversos indicadores, el grado de satisfacción de los estudiantes y la mejora del proceso de enseñanza-aprendizaje obtenida a través de los vídeos puestos a su disposición.

La elaboración de los videos corrió íntegramente de cuenta de los profesores que participan en el grupo. Los profesores pertenecen al Área de Máquinas y Motores Térmicos y son expertos en el programa de las asignaturas.

Uno de los principales retos del proyecto derivó del hecho de que los videos fueron concebidos como objetos de aprendizaje modulares y reutilizables (RLO, por sus siglas en inglés), es decir, un mismo video podría ser utilizado para cubrir los objetivos de aprendizaje de diferentes asignaturas y en distintas titulaciones.

Los RLO tienen entre sus principales características la brevedad, la flexibilidad para revisiones y/o actualizaciones, la compatibilidad con los formatos y dispositivos digitales más comunes y el hecho de que sean autocontenidos y tengan un carácter descontextualizado que facilite su reutilización.

El diseño y la elaboración de cada video fue responsabilidad de un único profesor, no obstante, al inicio y al final de cada una de las actividades del proyecto (ver Tabla 3) se llevaron a cabo reuniones entre todos los miembros del grupo en las que se tomaron decisiones colegiadas sobre ciertos aspectos que fueron considerados en todos los videos.

En concreto fue durante la fase de desarrollo (concretamente las actividades 1, 2 y 4) en la que se plantearon las discusiones relativas al enfoque de los contenidos, la adecuación al medio y la calidad técnica, entre otros, que dan lugar a este trabajo.

Tabla 3. Actividades incluidas en el proyecto docente

\begin{tabular}{ll}
\hline Actividad 1 & $\begin{array}{l}\text { Selección de la herramienta de captura de vídeo, diseño de la plantilla y formato de las } \\
\text { presentaciones incluidas en los vídeos }\end{array}$ \\
\hline Actividad 2 & Definición de los vídeos docentes a realizar y selección de la plataforma para su visionado \\
\hline Actividad 3 & $\begin{array}{l}\text { Preparación de cuestionarios para la evaluación de la mejora del proceso de enseñanza- } \\
\text { aprendizaje a través de los vídeos }\end{array}$ \\
\hline Actividad 4 & $\begin{array}{l}\text { Elaboración y utilización de los vídeos docentes como objetos de aprendizaje y lanzamiento de los } \\
\text { cuestionarios en Moodle2 }\end{array}$ \\
\hline Actividad 5 & $\begin{array}{l}\text { Compilación y análisis de los resultados. Planteamiento de acciones de mejora y análisis de } \\
\text { nuevas necesidades de innovación docente }\end{array}$ \\
\hline
\end{tabular}

\subsection{El enfoque de los contenidos}

La primera fase consistió en seleccionar los videos, las asignaturas en las que iban a ser utilizados y planificar su contenido.

El carácter RLO de los videos desarrollados exige planificar el contenido del video desde una perspectiva más amplia, que recoja los objetivos de aprendizaje comunes de las

(cc) BY-NC-ND 2018, Universitat Politècnica de València

Congreso IN-RED (2018) 
asignaturas en las que ser utilizados en un alto porcentaje y teniendo constancia de que el resto de los objetivos podían ser completados por otras actividades que reflejaran las especificidades de cada asignatura.

Tomando como base las guías docentes de todas las asignaturas, cada profesor responsable de una asignatura en las que se iba a utilizar un video en concreto elaboró un listado de los objetivos de aprendizaje esperados.

El profesor desarrollador del video planteo una propuesta de desarrollo del video y en una de las reuniones de coordinación se comprobó que se cubrieran todos los objetivos de aprendizaje comunes.

No se dio ningún caso en el que no fueran cubiertos; que todos los profesores pertenecieran a la misma área de conocimiento y tuvieran experiencia docente en todas las asignaturas para las que iban a ser utilizados facilitó la tarea.

En los casos en que la propuesta cubrió más objetivos que los comunes se discutió sobre la idoneidad de mantenerlos o no, optando en la mayoría de los videos por mantenerlos.

\subsection{La adecuación al medio}

Aunque existen diferencias entre la comunicación virtual y la presencial, de la misma forma que en el aula, aunque acrecentado cuando el canal de comunicación es la pantalla de su ordenador, uno de los mayores desafíos para la elaboración de videos docentes es atraer y mantener el interés de los estudiantes.

Hay ciertas técnicas que pueden utilizarse para lograr y mantener el interés de los estudiantes en la creación de videos educativos y de capacitación de alta calidad como, por ejemplo, insertar materiales complementarios a lo largo del visionado como preguntas, cuestiones o ejercicios cortos que pueden ayudar a los estudiantes a percibirlo como una experiencia de aprendizaje más auténtica. Por ello, se está trabajando para incorporar en cada video, cuestionarios que permitan al estudiante su auto-evaluación y sirvan para reforzar los objetivos de aprendizaje planteados.

La duración del video también juega en contra de mantener dicha atención. Los videos deben ser relativamente cortos. Se decidió que la duración media debería de ser de 10 minutos y en el caso de contenidos más largos se hicieron divisiones en partes.

Otra forma de conectarse con los estudiantes es que aparezca la imagen del profesor en el video. Es más fácil que los estudiantes establezcan una conexión cuando los alumnos ven al profesor presentando en persona el contenido. No obstante, dado que los videos eran para una disciplina técnica, se decidió favorecer la atención a otras animaciones y videos y prescindir de la imagen del profesor si bien estaba presente como narrador.

Finalmente, para romper la monotonía, se optó por que los videos fueran ligeramente diferentes entre sí: unos con diapositivas, otros con videoclips, otros con grabación de pantalla (screencasts), etc.

\subsection{La calidad técnica}

(c)) EY-NG-ND 2018, Universitat Politècnica de València

Congreso In-Red (2018) 
No puede perderse de vista que los estudiantes actuales poseen expectativas muy altas respecto a los productos audiovisuales por lo que se prestó atención especial a la calidad técnica de los videos.

A partir de la revisión realizada sobre ventajas y limitaciones de las distintas herramientas informáticas disponibles para la captura y edición de vídeos, los profesores participantes en este proyecto decidieron utilizar la herramienta Camtasia debido a que sus funcionalidades permiten crear vídeos de gran calidad técnica en formato mp4 de una manera sencilla, en contraste con otras herramientas gratuitas más limitadas desde el punto de vista técnico.

Dicho software tiene buena una buena capacidad tanto de edición de video como de audio.

Los videos requieren una preparación diferente a la de una clase en vivo. En primer lugar, hay que decidir si hablar desde un script o libremente. En el primer caso, debe de sonar como cuando se habla, por lo que hay que comprobarlo leyendo en voz alta mientras se desarrolla. Con cualquiera de las dos opciones, se debe de ensayar con anterioridad d la grabación para que las palabras sean correctas y suenen naturales. El tono debe de ser confiado y optimista, no agresivo, cansado, impaciente o aburrido. El momento de la grabación debe de elegirse con cuidado, evitando momentos en los que la voz aparezca cansada.

Muchos expertos sostienen que la calidad del audio importa mucho más que la calidad de video. Tras la fase de evaluación se detectaron problemas con el sonido en algún video por lo que va a volver a grabarse el audio con un micrófono de configuración direccional.

Para dar homogeneidad y consistencia a los vídeos docentes, se diseñó una plantilla común de PowerPoint con formatos establecidos para la portada, el índice de contenidos, los títulos y subtítulos de las distintas secciones, el tipo de letra y el fondo, teniendo en cuenta las recomendaciones generales recogidas en la bibliografía especializada.

- Utilizar dos o tres tipos de video como máximo (con una imagen fija contando dos

- Utilizar uno o como máximo dos tipos de transición dentro de cada video, preferentemente simples.

- Utilizar solo un color principal y uno o máximo dos fuentes por video (una principal y otra para acentuar conceptos).

- Elegir colores que sean fáciles para los ojos (p.ej. el azul) y fuentes que sean fácilmente legibles.

Por otra parte, dentro de esta actividad se incluyó una revisión de los tipos de licencias Creative Commons (CC) y las distintas posibilidades de reconocer la autoría de los vídeos docentes realizados, optándose finalmente por utilizar la licencia CC BY NC ND.

\section{Resultados}

El resultado de las decisiones tomadas por el equipo de profesores con respecto a los aspectos metodológicos presentados en la anterior sección, fueron validaron con las 
respuestas de los estudiantes recopiladas a través de los cuestionarios intermedios (Zabalza et al., 2017).

Dicho cuestionario consiste en 5 preguntas en una escala Likert de 1 a 5 , con las que los estudiantes establecieron la valoración de cada video utilizado atendiendo a cinco características:

1. Amenidad y capacidad de motivación

2. Adecuación de la duración del vídeo

3. Utilidad para resolver dudas y asimilar contenidos

4. Calidad técnica del vídeo

5. Valoración global del vídeo

La cuestión 3 está relacionado con el aspecto "Enfoque de los contenidos”, las cuestiones 1 y 2 con “Adecuación al medio” y la cuestión 4 está directamente relacionada con el aspecto del mismo nombre.

En la figura 1 se muestra la distribución de las respuestas dadas por el mismo grupo de estudiantes para el conjunto de los vídeos utilizados (19) en una de las asignaturas. Se observa que más del $40 \%$ de los estudiantes otorga una puntuación de 4 puntos o más (en una escala del 1 al 5) en los aspectos de amenidad, duración, utilidad para la resolución de dudas y calidad técnica de los videos docentes. Por otro lado, a pesar de haberse limitado, la duración del video es considerada como el aspecto peor valorado. Asimismo, el 50\% de los estudiantes asigna una puntuación igual o superior a los 4 puntos como calificación global de los videos pudiéndose concluir que la mayoría de los estudiantes han quedado muy satisfechos con los videos utilizados.

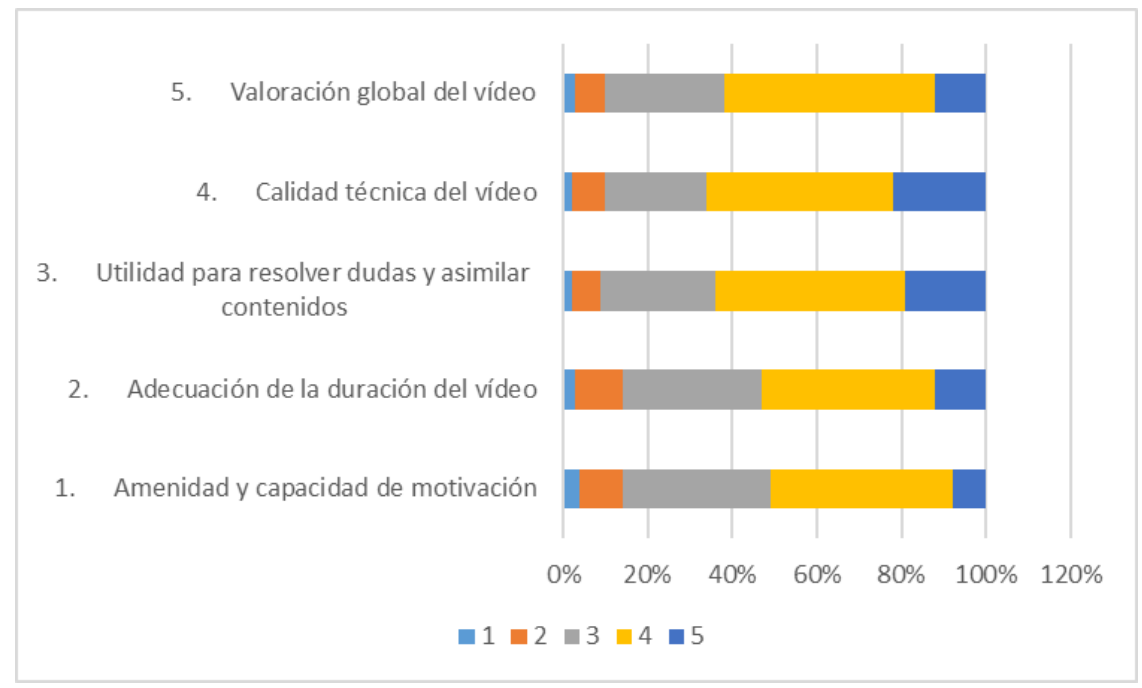

Fig. 1 Valoración de los aspectos metodológicos por parte de los estudiantes

En cuanto a la mejora de las calificaciones obtenida por el mismo alumnado en la prueba de evaluación de la asignatura, tal como muestra la Figura 2, se puede observar como en el curso 2015-16 aumentaron 10 puntos las calificaciones en la horquilla de 8 a 9, y en 9 
puntos las calificaciones en la zona de sobresaliente, reduciéndose las calificaciones más bajas. Esta mejora queda también manifiesta en un aumento de la nota promedio en 0.25 puntos.

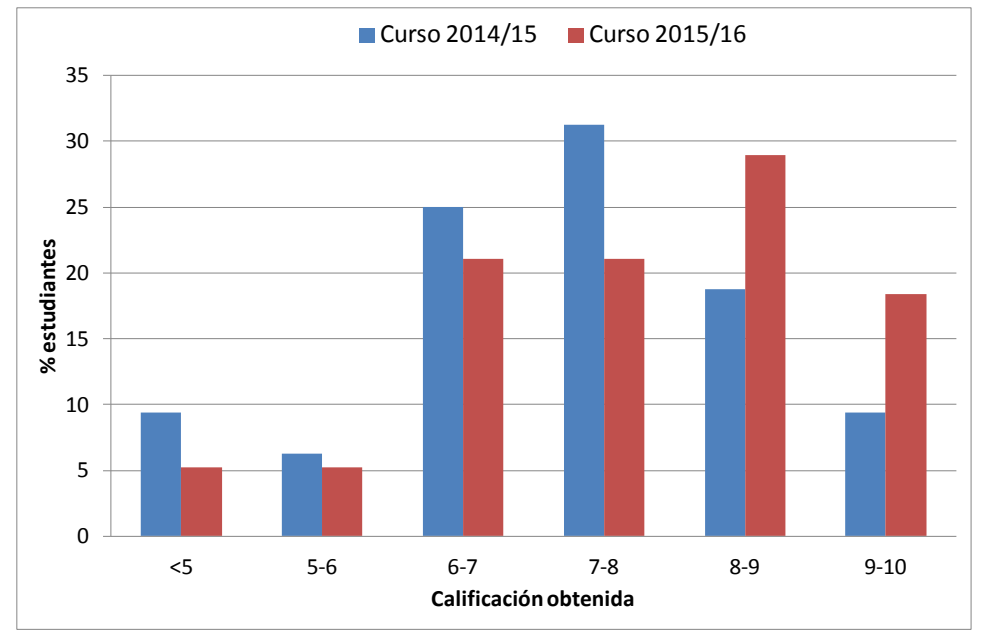

Fig. 2 Comparativa de las calificaciones obtenidas en las prácticas de la asignatura analizada en los cursos académicos 2014-15 y 2015-16

Finalmente, queda por comprobar en qué grado la efectividad de los videos en la mejora del proceso del aprendizaje se debe a los aspectos inherentes a su elaboración y no solo a la predisposición del estudiante o a la habilidad del profesor.

Dado que la predisposición del estudiante es un aspecto difícil de medir, sobre todo cuando se pretende comparar resultados de dos grupos de estudiantes, para responder a la cuestión se han analizado las calificaciones obtenidas a lo largo de todo el proceso de evaluación de un único grupo de estudiantes, en concreto el grupo de 70 estudiantes de la asignatura "Máquinas y motores térmicos”.

Dicha asignatura posee un sistema de evaluación continua que con el fin de cumplir con el Reglamento de Normas de Evaluación del Aprendizaje de la Universidad de Zaragoza coexiste con la evaluación global.

La Tabla 4 resume el conjunto de pruebas que constituyen la evaluación continua de la asignatura con una breve descripción de su realización y criterios, así como su peso sobre la calificación final del estudiante en la asignatura. Estas pruebas se encuentran alineadas con las tres actividades principales que se desarrollan en la asignatura (clases, prácticas y trabajo), las competencias genéricas que son evaluadas y los resultados de aprendizaje previstos.

Los dos videos utilizados en la asignatura según se recoge en la Tabla 2 se incluyeron dentro de 2 de las 5 actividades desarrolladas como "Prácticas de laboratorio". En concreto, los videos "Ciclos de potencia con turbina de gas-Brayton” y "Motores Térmicos” debieron de ser visionados por los alumnos antes de realizar la práctica.

(cc) EY-NC-ND 2018, Universitat Politècnica de València

Congreso IN-RED (2018) 
Tabla 4. Sistema de evaluación de la asignatura "Máquinas y Motores Térmicos"

\begin{tabular}{|l|l|c|}
\hline Instrumento & Realización/criterios & Peso \\
\hline \multirow{2}{*}{$\begin{array}{l}\text { Examen: prueba escrita } \\
\text { individual de teoría y problemas }\end{array}$} & $\begin{array}{l}\text { Cuestiones teóricas y/o teórico-prácticas: } \\
\text { Diez cuestiones teórico-prácticas } \\
\text { orientadas a conceptos, definiciones, etc. } \\
\text { Se evalúan conocimientos teóricos }\end{array}$ & $\begin{array}{l}19.5 \% \\
\text { (30\% de 65\%) }\end{array}$ \\
\cline { 2 - 3 } & $\begin{array}{l}\text { Problemas: 2 problemas de media o larga } \\
\text { extensión. Se evalúa la capacidad de } \\
\text { aplicar conocimientos a la práctica y } \\
\text { capacidad de análisis }\end{array}$ & $\begin{array}{c}45.5 \% \\
\text { (70\% de 65\%) }\end{array}$ \\
\hline $\begin{array}{l}\text { Prácticas de laboratorio: prueba } \\
\text { escrita }\end{array}$ & $\begin{array}{l}\text { Informe escrito según directrices del } \\
\text { guión. Se evalúa la aplicación de los } \\
\text { conocimientos teóricos }\end{array}$ & $15 \%$ \\
\hline Trabajo de asignatura & $\begin{array}{l}\text { Se propone un estudio del caso para } \\
\text { realizar en equipo. La documentación } \\
\text { puede estar en inglés. Se evalúa por } \\
\text { medio de un entregable, una tutoría de } \\
\text { control y la defensa oral del trabajo }\end{array}$ & $20 \%$ \\
\hline
\end{tabular}

Con el fin de identificar el efecto del uso de los videos, se separaron las calificaciones de las actividades en las que se habían utilizado los videos de las actividades en las que no. Se calcula el promedio de las calificaciones "no vinculadas" con los videos asumiendo que pueden ser un buen indicador de la predisposición del estudiante, de forma que la diferencia de las calificaciones de las dos prácticas con dicho valor promedio y dado que el profesor también es el mismo puede ser únicamente atribuible a los aspectos relacionados con la elaboración de los videos.

Las figuras 3 y 4 resumen los resultados obtenidos para las 40 observaciones válidas y permiten concluir sobre la influencia positiva del uso de videos. En la Figura 3 se muestra la estadística de las calificaciones no influidas directamente por los videos mientras que la Figura 4 muestra la misma estadística para las calificaciones de las dos prácticas.

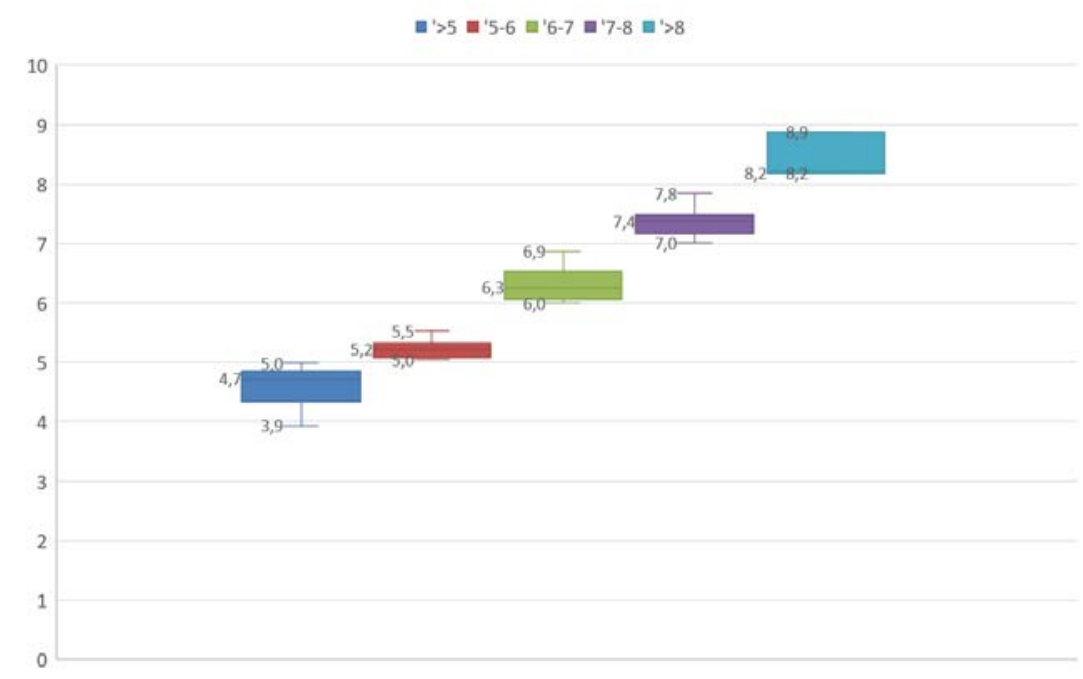

Fig. 3 Distribución de las calificaciones obtenidas en las actividades no vinculadas a los videos docentes 


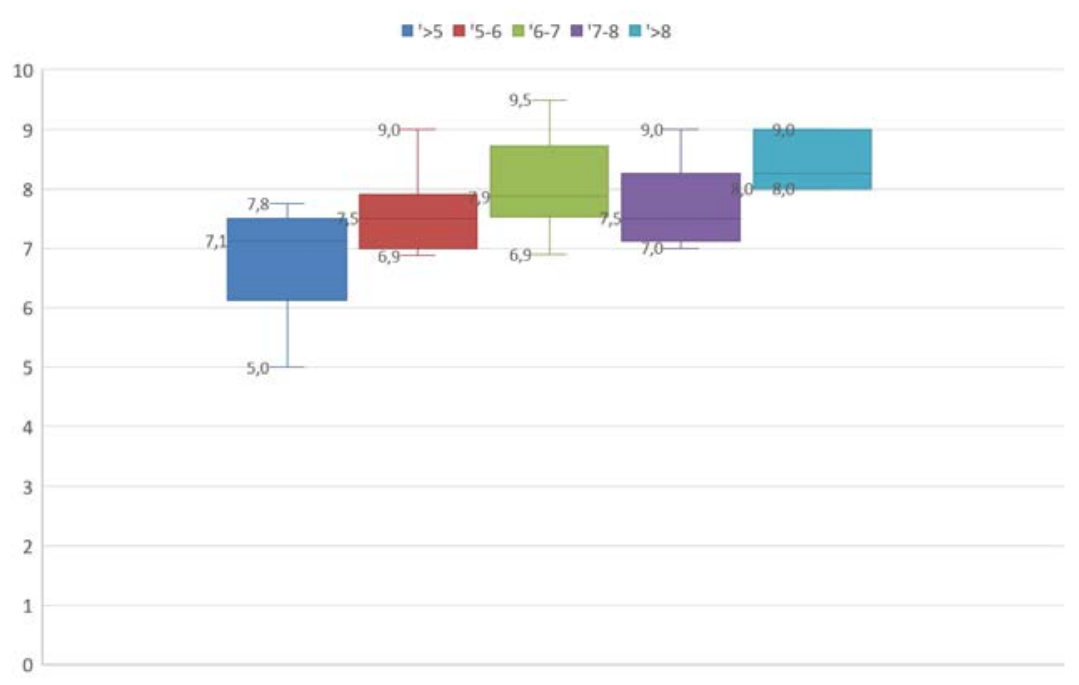

Fig. 4 Distribución de las calificaciones obtenidas en las actividades vinculadas a los videos docentes

Se observa que las notas de las actividades sujetas al proyecto de innovación (Figura 4) son en general superiores a las del resto de las actividades docentes (Figura 3). El incremento en la nota es apreciablemente mayor en el caso del $79 \%$ de los estudiantes que son los que obtienen notas por debajo del notable (las medias pasan de 4.7 a 7.1, de 5.2 a 7.5 y de 6.3 a 7.9) mientras que en los grupos con mejores notas no se observa una incidencia tan clara. Como consecuencia de ello, las calificaciones de las actividades apoyadas con los videos muestran una distribución más homogénea entre los estudiantes.

Todo lo anterior permite concluir que hay evidencias de que el uso de los videos docentes tal y como fueron diseñados en el proyecto que se describe han cumplido con las expectativas con las que fueron planteados. Asimismo, puede concluirse que son los estudiantes con niveles medios y bajos de predisposición al aprendizaje los que encuentran un mayor beneficio en este tipo de herramientas docentes lo que impulsa a los autores a continuar incorporando este tipo de materiales al resto de actividades docentes de las asignaturas.

\section{Conclusiones}

Crear videos docentes de alta calidad requiere mucho más trabajo que preparar una sesión presencial, ya que además de dominar la materia, supone un desafío creativo para el que el profesor se encuentra en ocasiones limitado. La creación de grupos de trabajo o el apoyo de unidades audiovisuales en los centros puede facilitar dicha tarea.

Invertir más tiempo en la fase de desarrollo no necesariamente hace que los videos sean mejores desde una perspectiva pedagógica, ya que deben de ser complementados con otras actividades y metodologías, pero probablemente facilitan los resultados.

Además, la buena aceptación que han tenido por parte de los estudiantes y la constancia expresa de que les ha ayudado en su proceso de aprendizaje, motivan a los profesores a

(cc) EY-NC-ND 2018, Universitat Politècnica de València

Congreso IN-RED (2018) 
continuar en la aplicación de metodologías docentes que hagan uso de los videos producidos, a mejorarlos paulatinamente desde la experiencia adquirida y a crear nuevos.

Asimismo, a partir del análisis de los resultados obtenidos por una muestra de los estudiantes involucrados en el proyecto docente, se demuestra cómo un adecuado enfoque de los contenidos, la adaptación al medio visual y la calidad técnica de los videos, contribuyen a su efectividad en el proceso de enseñanza-aprendizaje.

\section{Agradecimientos}

Este trabajo ha sido financiado por medio de los proyectos de innovación docente PIIDUZ_17_299 y PIIDUZ_17_165 (Programa de Proyectos de Innovación Docente para Grupos de Profesores del Vicerrectorado de Política Académica de la Universidad de Zaragoza).

\section{Referencias}

Kearney, M., Jones, G., \& Roberts, L. (2012). An emerging learning design for student-generated 'iVideos'. In C. Alexander, J. Dalziel, J. Krajka \& E. Dobozy (Eds.), Teaching English with Technology, Special Edition on LAMS and Learning Design volume 3, 12(2), 103-120, http://www.tewtjournal.org.

Tereza Angelova Stefanova, Using of Training Video Films in the Engineering Education, Procedia Social and Behavioral Sciences, Volume 116, 2014, Pages 1181-1186, ISSN 1877-0428, https://doi.org/10.1016/j.sbspro.2014.01.366.

ZABALZA, I., PEÑA, B., LLERA, E.M., USÓN, S., MARTÍNEZ, A. y ROMEO, L.M. (2017). "Evaluación de la mejora del proceso de enseñanza-aprendizaje mediante la integración de objetos de aprendizaje reutilizables en un curso abierto OCW” en Congreso In-Red 2017, 13 y 14 de julio de 2017. Valencia.

Willmot, P., Bramhall, M., \& Radley, K. (2011). Introducing audio-visual media for inspirational learning and positive engagement, SEFI. International Conference on Engineering Education, Lisbon, Portugal, September 2011, Lisbon, SEFI, 420-426.

Cole, A. W.; Allen, M.; Anderson, C.; Bunton, T.; Cherney, M. R.; Draeger, Jr., R.; Featherston, M.; Fisher, V. C.; Motel, L.; Nicolini, K. M. \& Peck, B. (2017). Student predisposition to instructor feedback and perceptions of teaching presence predict motivation toward online courses. Online Learning, 21(4), 245-262. doi: 10.24059/olj.v21i4.966

Sujo de Montes, L.E. \& Gonzales, C.L. (2000). More than Having a Connection: Qualitative Factors that Affect Learning in a Web-based University Course. In D. Willis, J. Price \& J. Willis (Eds.), Proceedings of SITE 2000--Society for Information Technology \& Teacher Education International Conference (pp. 177-182). Chesapeake, VA: Association for the Advancement of Computing in Education (AACE). Retrieved March 25, 2018 from https://www.learntechlib.org/p/15549/.

PEÑA, B., ZABALZA, I., USÓN, S., LLERA, E.M., MARTÍNEZ, A. y ROMEO, L.M. (2017). "Experiencia piloto de aula invertida para mejorar el proceso de enseñanza-aprendizaje en la asignatura de Termodinámica Técnica” en Congreso In-Red 2017, 13 y 14 de julio de 2017. Valencia. 